\title{
Confiabilidade do Perfil de Saúde de Nottingham após Acidente Vascular Encefálico
}

\author{
Reliability of the Nottingham Health Profile after suffering a stroke
}

Dinalva Lacerda Cabral ${ }^{1}$

Caroline Guimarães Damascena ${ }^{1}$

Luci Fuscaldi Teixeira-Salmela ${ }^{2}$

Glória Elizabeth Carneiro Laurentino ${ }^{1}$

${ }^{1}$ Departamento de Fisioterapia, Universidade Federal de Pernambuco. Av. Jornalista Anibal Fernandes s/n, Cidade Universitária. 50740-560 Recife PE. gloriaecl@ufpe.br ${ }^{2}$ Departamento de Fisioterapia, Universidade Federal de Minas Gerais.

\begin{abstract}
This article seeks to evaluate the reliability, internal consistency and accuracy of the Nottingham Health Profile (NHP), namely a quality of life $(Q L)$ instrument for individuals after suffering a stroke. This cross-sectional study was carried out in the communities of Recife in the State of Pernambuco. The sample was composed of 53 individuals at the chronic stroke phase. After checking the cognitive state, the NHP was applied. Descriptive statistics were employed for characterization of the sample; Cronbach's alpha $(\alpha)$ coefficients were used for evaluation of internal consistency, and intra-class correlation coefficients (ICC) were used for investigation of reliability, as well as consistency of the Bland and Altman plotting tests with 5\% level of significance. Their perceptions of their own health were $83.3 \%$ positive for the majority of NHP criteria (average scores $>$ 50 points), except for the "physical ability" criterion where scores ranged between 41.5 and 50 points. The NHP had good internal consistency with $\alpha$ values between 0.81 to 0.87 ; excellent reliability indices for the "pain" and "physical ability" criteria (ICC > 0.90); and 95\% consistency. Besides being simple and easily applied, NHP had adequate clinimetric properties for the assessment of individuals after a chronic stroke.
\end{abstract}

Key words Nottingham health profile, Reliability, Quality of life, Stroke
Resumo O artigo tem o objetivo de avaliar a confiabilidade, a consistência interna e a concordância do instrumento de qualidade de vida (QV) Perfil de Saúde de Nottingham (PSN) em indivíduos após Acidente Vascular Encefálico (AVE). Estudo do tipo transversal realizado em comunidades da Cidade do Recife-PE. A amostra foi composta por 53 indivíduos na fase crônica do AVE. Após verificação do estado cognitivo foi aplicado o PSN. Utilizou-se estatística descritiva para caracterizar a amostra; a consistência interna foi medida através do alfa $(\alpha)$ de Cronbach; a confiabilidade com o coeficiente de correlação intraclasse (CCI) e a concordância pelo teste de plotagem Bland and Altman, com nível de significância de 5\%. A percepção dos indivíduos sobre suas saúdes teve uma tendência positiva em 83,3\% dos domínios do PSN (média > 50 pontos), exceto para o dominio "habilidades físicas" que variou entre 41,5 e 50 pontos. $O$ instrumento obteve boa consistência interna, com $\alpha$ entre 0,81 e 0,87; excelente confiabilidade nos dominios "dor" e "habilidades físicas", ICC >0,90 ( $p$ $<0,01$ ); e concordância em 95\% das ocasiões. O PSN, além de ser um instrumento simples e de fácil aplicação, apresentou propriedades clinimétricas adequadas para avaliar a $Q V$ de indivíduos pósAVE na fase crônica.

Palavras-chaves Perfil de saúde de Nottingham, Confiabilidade, Qualidade de vida, Acidente Vascular Encefálico 


\section{Introdução}

Segundo a Organização Mundial de Saúde (OMS), no ano de 2001, mais de 20 milhões de indivíduos sofreram Acidente Vascular Encefálico (AVE), dos quais, 5,5 milhões foram a óbito' ${ }^{1}$. Embora as taxas de sobrevivência sejam elevadas, cerca de $90 \%$ dos acometidos desenvolvem algum tipo de deficiência, o que faz desta patologia a primeira causa de incapacidade funcional no ocidente $^{2,3}$. No Brasil, 250 mil novos casos surgem a cada ano e $30 \%$ destes permanecem com sequelas, como a hemiplegia ou hemiparesia ${ }^{4}$. Assim, preservar a qualidade de vida $(\mathrm{QV})$ no pós-AVE é imprescindível para que a pessoa possa superar o quadro clínico e emocional instaurado 5 .

A OMS definiu a QV como "a percepção do indivíduo de sua posição na vida, no contexto da cultura e sistemas de valores nos quais ele vive e em relação aos seus objetivos, expectativas, padrões e preocupações" ${ }^{\text {. }}$ Na literatura, tem-se usado o termo QV relacionada à saúde (QVRS) como a percepção do indivíduo quanto ao seu estado físico, funcional, emocional e social ${ }^{7,8}$. Portanto, a avaliação da QVRS torna-se relevante para mensurar o impacto de doenças na vida dos indivíduos, bem como para contribuir no planejamento de ações, previsão e alocação nos serviços de saúde ${ }^{8}$.

O Perfil de Saúde de Nottingham (PSN) é um instrumento genérico de QVRS que, em 2004, foi traduzido e adaptado para a população brasilei$\mathrm{ra}^{8}$. A versão brasileira do PSN foi desenvolvida em portadores de doenças crônicas, apresentando alto índice de validade clínica ${ }^{8}$. Entretanto, estudos referem ${ }^{9,10}$ que instrumentos genéricos podem subestimar o impacto de uma condição de saúde, como o AVE, pois, provavelmente, são menos sensíveis para explorar os efeitos incapacitantes da doença. Dessa forma, faz-se necessário um número maior de investigações com aplicabilidade do PSN para melhor assegurar suas propriedades clinimétricas e caracterizar suas medidas como confiáveis e válidas após-AVE ${ }^{11}$.

Até o presente momento, não foram encontrados estudos que analisassem as propriedades clinimétricas da versão brasileira do PSN exclusivamente em indivíduos pós-AVE. Nesta perspectiva, objetivou-se avaliar a confiabilidade (testereteste e interexaminadores) e a consistência interna do PSN, versão brasileira, em indivíduos comunitários na fase crônica pós-AVE; bem como verificar a concordância das medidas obtidas pelo mesmo examinador (teste-reteste) e por diferentes examinadores (interexaminadores).

\section{Materiais e métodos}

Trata-se de um estudo do tipo corte transversal, realizado em seis comunidades vinculadas a unidades de saúde pertencentes ao Distrito Sanitário IV da Cidade do Recife (PE), Nordeste do Brasil, no período de outubro/2009 a março/ 2010. A inclusão da Estratégia de Saúde da Família - ESF e do Programa de Agentes Comunitários de Saúde - PACS para a seleção da amostra, deveu-se ao fato destes atuarem em território adstrito, realizando cadastramento domiciliar e diagnóstico situacional de saúde, facilitando a identificação dos casos e o enquadramento nos critérios de inclusão da pesquisa.

\section{Cálculo Amostral e Critérios de Elegibilidade}

Para calcular a amostra, inicialmente foi realizado um estudo piloto com 30 indivíduos na fase crônica pós-AVE. A partir dos resultados obtidos nos seis domínios do PSN, calculou-se, então, a amostra necessária para a estimativa de uma média considerando 95\% de confiança. Assim, admitindo um erro máximo de 10 pontos, o tamanho amostral foi estimado em 53 indivíduos.

Para inclusão no estudo, os indivíduos, de ambos os sexos, deveriam ter o diagnóstico de AVE, isquêmico ou hemorrágico, primário ou recorrente, há mais de seis meses (fase crônica da doença) e confirmado nos prontuários médicos ou fichas cadastrais das unidades de saúde; ter idade superior a 20 anos e competência mental, avaliada através da versão brasileira do mini exame do estado mental (MEEM) ${ }^{12}$. Foram excluídos os indivíduos com déficits auditivo e/ou distúrbios da fala que pudessem comprometer a comunicação entre os interlocutores e aqueles que, durante o período da pesquisa, relataram fatos que poderiam alterar a $\mathrm{QV}$ como a ocorrência de quedas e/ou perda de um ente querido.

\section{Instrumentos e Coleta de Dados}

Cada voluntário foi entrevistado individualmente e durante visitas domiciliares, com tempo médio de 30 minutos para cada entrevista. Inicialmente, foram coletados os dados socioeconômicos, demográficos e clínicos, por meio de uma ficha de avaliação padronizada.

Para categorização da QVRS, os indivíduos foram avaliados por meio do PSN que fornece respostas no formato "sim"/"não". Este instrumento é composto por 38 itens, agrupados em 
seis domínios: "nível de energia” (NE), 3 itens avalia o nível de energia e de fadiga; “dor" (D), 8 itens - avalia a presença de dor, sua intensidade e sua interferência nas atividades de vida diária (AVD); "reações emocionais" (RE), 9 itens - apresenta questões sobre ansiedade, depressão, alterações no comportamento ou descontrole emocional e bem-estar psicológico; "interação social" (IS), 5 itens - analisa a existência do sentimento de solidão e a dificuldade de interagir com outras pessoas; "habilidades físicas" (HF), 8 itens - analisa a presença de limitações durante a realização das AVD e "sono" (S), 5 itens - avalia a qualidade do sono e a presença de insônia (13). Cada percepção de saúde positiva corresponde a 1 (um) e negativa corresponde a 0 (zero), perfazendo uma pontuação máxima igual a $38^{8}$. O PSN, embora seja autoadministrado, foi aplicado por examinadores devidamente treinados, no intuito de evitar interferência dos resultados devido à possível heterogeneidade nos graus de instrução da amostra, conforme recomendado por Teixeira-Salmela et al. ${ }^{8}$.

Para avaliação da confiabilidade teste-reteste, cada examinador (A e B) aplicou o PSN em duas ocasiões diferentes (teste 1 e 2), respeitando-se um prazo mínimo de sete dias entre as avaliações para diminuir a possibilidade de memorização dos resultados. E para a avaliação da confiabilidade interexaminadores, o PSN foi aplicado pelo examinador "A" e "B" em momentos distintos. Nenhum dos examinadores esteve presente durante a entrevista realizada pelo outro examinador e, após a avaliação, não houve acesso aos dados coletados pelo outro, evitando trocas de informações. No período de tempo entre cada avaliação, os indivíduos que relataram algum fato que pudesse interferir na confiabilidade das medidas como quedas e/ou perda de um ente querido, foram automaticamente excluídos do estudo.

\section{Análise Estatística}

Para caracterizar a amostra e a distribuição dos escores obtidos, utilizou-se estatística descritiva (média e desvio padrão) e, quando pertinente, os intervalos de confiança (IC) foram calculados. A quantificação da pontuação total do PSN e dos seis domínios foi obtida a partir da soma do número de respostas "não" e realizado um cálculo de proporção para transformar o valor em porcentagem. Dessa forma, quanto mais próxima de 0 (zero) foi a porcentagem obtida, pior a percepção de saúde; e quanto mais próxima de 100 (cem), melhor ${ }^{13}$.
Para cada domínio do PSN, avaliou-se a confiabilidade teste-reteste e interexaminadores através do coeficiente de correlação intra-classe (CCI), cujos valores variam de 0 (zero) a 1 (um) e pode ser classificado como inaceitável $(\leq 0,70)$, aceitável (entre 0,71 e 0,79), muito bom (entre $0,80$ e 0,89$)$ e excelente $(\geq 0,90)^{14}$.

A concordância teste-reteste (examinador A - testes 1 e 2) e interexaminadores (examinadores A e B - teste 1) do escore total do PSN foi mensurada pelo teste de plotagem Bland and Altman, onde foram construídos diagramas de dispersão mostrando as diferenças individuais (no eixo y) em função das médias observadas nas duas avaliações (no eixo $\mathrm{x}$ ). Considerando-se que ao aplicar PSN em momentos distintos, aos mesmos indivíduos e em situações semelhantes, as médias devem se aproximar do valor 0 (zero) ${ }^{15}$.

Para a confiabilidade do tipo consistência interna, utilizou-se o alfa $(\alpha)$ de Cronbach. O coeficiente varia de 0 (zero) a 1 (um), classificando a propriedade como muito boa $(>0,90)$, boa (entre 0,80 e 0,90 ), razoável (de 0,70 a $<0,80$ ), fraca (de 0,60 a $<0,70)$ e inadmissível $(<0,60)^{16}$.

Os softwares utilizados para análise dos dados foram o Excel ${ }^{\circledR}$ 2000, o SPSS (Statistical Package for Social Sciences) para Windows ${ }^{\circledast}$ - versão 13.0 e o Prisma - versão 4, considerando 95\% de confiança.

\section{Considerações Éticas}

O estudo foi aprovado pelo Comitê de Ética em Pesquisa do hospital Agamenon Magalhães, conforme resolução $n^{\circ}$. 196/96 do Conselho Nacional de Saúde (CNS) ${ }^{16}$. Todos os indivíduos que concordaram em participar da pesquisa, mediante a explicação dos objetivos, assinaram o termo de consentimento livre e esclarecido.

\section{Resultados}

A Figura 1 mostra que um total de 108 voluntários foram inicialmente contatados através de visita domiciliar. Destes, 55 foram excluídos por não atenderem aos critérios de elegibilidade, atingindo-se assim o número de 53 indivíduos previamente estimados durante o cálculo amostral. O PSN foi aplicado em quatro momentos distintos, perfazendo um total de 267 visitas domiciliares.

De acordo com os dados sociodemográficos, $55 \%$ dos indivíduos avaliados eram mulheres, as idades variaram de 42 a 85 anos, com média de 66 anos $( \pm 9,9), 60 \%$ tinha o ensino fundamental 


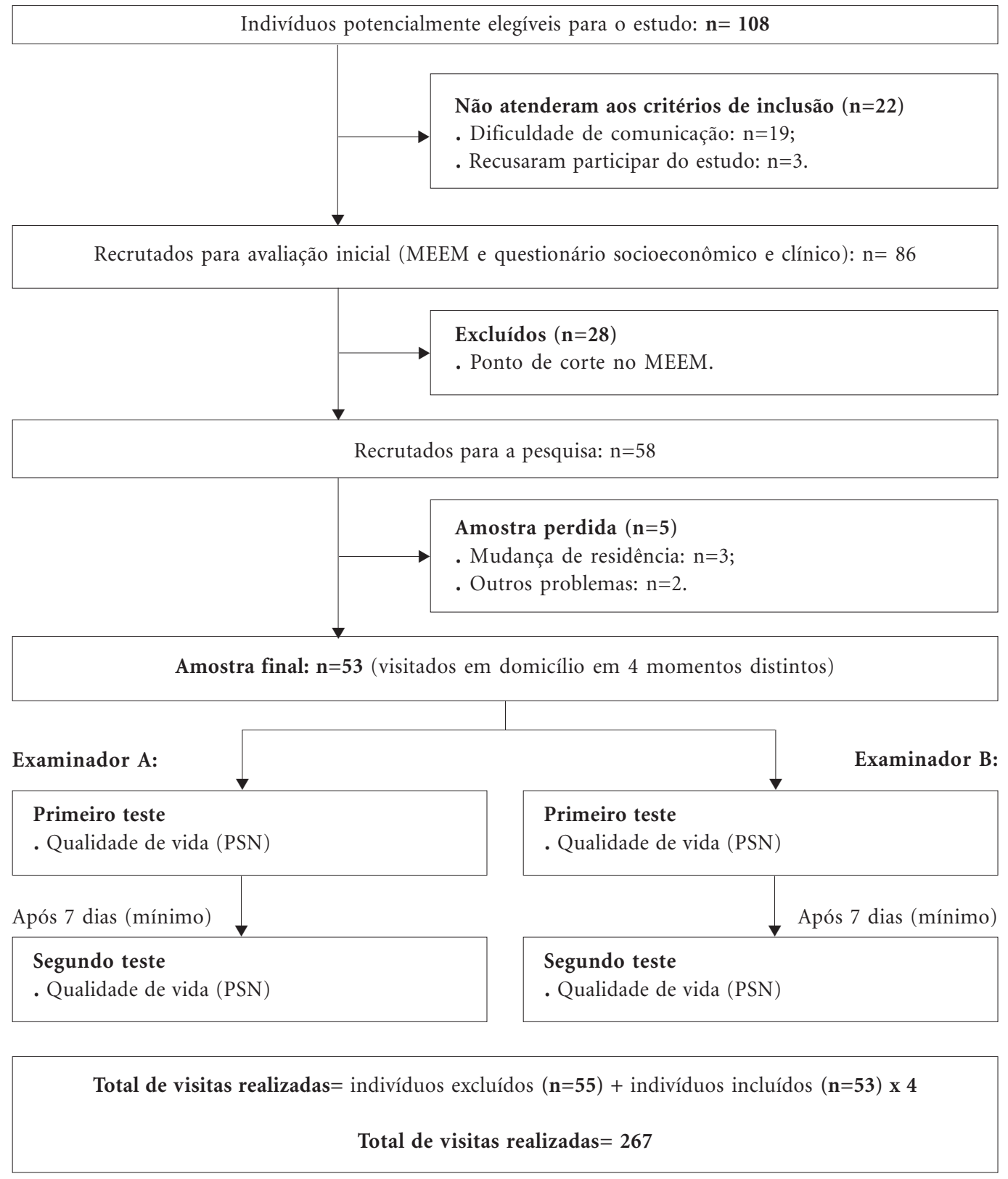

MEEM= mini exame do estado mental; AVE= Acidente Vascular Encefálico; PSN= perfil de saúde de Nottingham.

Figura 1. Fluxograma de seleção da amostra estudada, Recife-PE, Brasil, 2010.

e $57 \%$ com renda familiar per capita $\leq 1 / 2$ salário mínimo. Os dados clínicos revelaram que 53\% da amostra apresentou o hemicorpo esquerdo acometido pelo AVE, todos os entrevistados eram portadores de hipertensão arterial sistêmica (HAS), 91\% eram sedentários, 89\% tinham acom- panhante para auxiliar nas suas AVDs e 75,5\% não fazia tratamento fisioterapêutico (Tabela 1).

No escore total e em 83,3\% domínios do PSN, a pontuação foi acima de 50 pontos, exceto para o domínio "habilidades físicas" que apresentou os menores valores (entre 41,5 e 50 pontos). Ana- 
Tabela 1. Características sócio-demográficas e clínicas de indivíduos pós AVE residentes em comunidades da Cidade do Recife-PE, Brasil, 2010.

\begin{tabular}{|c|c|}
\hline Variável & \\
\hline \multicolumn{2}{|l|}{ Sexo $\mathrm{n}(\%)$} \\
\hline Masculino & $24(45,0)$ \\
\hline Feminino & $29(55,0)$ \\
\hline Idade (média em anos \pm DP) & $(66 \pm 9,9)$ \\
\hline \multicolumn{2}{|l|}{ Grau de escolaridade n (\%) } \\
\hline Analfabeto & $10(19,0)$ \\
\hline Alfabetizado ${ }^{a}$ & $1(2,0)$ \\
\hline Ensino fundamental & $32(60,0)$ \\
\hline Outros & $10(19,0)$ \\
\hline \multicolumn{2}{|l|}{ Estado civil n (\%) } \\
\hline Casado & $18(34,0)$ \\
\hline Solteiro & $11(21,0)$ \\
\hline Viúvo & $16(30,0)$ \\
\hline Outros & $8(15,0)$ \\
\hline \multicolumn{2}{|l|}{ Ocupação atual n (\%) } \\
\hline Aposentado & $36(68,0)$ \\
\hline Pensionista & $4(7,0)$ \\
\hline Outras & $13(25,0)$ \\
\hline \multicolumn{2}{|l|}{ Renda familiar per capita n (\%) } \\
\hline$\leq 1 / 2$ salário mínimo & $30(57,0)$ \\
\hline 1/2 a 1 salário mínimo ${ }^{\mathrm{b}}$ & $12(23,0)$ \\
\hline > 1 salário mínimo & $6(11,0)$ \\
\hline Não soube informar & $5(9,0)$ \\
\hline \multicolumn{2}{|l|}{ Chefe da família n (\%) } \\
\hline Sim & $37(70,0)$ \\
\hline Não & $16(30,0)$ \\
\hline \multicolumn{2}{|l|}{ Episódios de AVE n (\%) } \\
\hline $\mathrm{Um}$ & $37(70,0)$ \\
\hline Mais de um & $16(30,0)$ \\
\hline Tempo pós AVE (média em anos \pm DP) & $(6,0 \pm 7,0)$ \\
\hline \multicolumn{2}{|l|}{ Hemicorpo acometido n (\%) } \\
\hline Direito & $25(47,0)$ \\
\hline Esquerdo & $28(53,0)$ \\
\hline \multicolumn{2}{|l|}{ Doenças associadas n (\%) } \\
\hline HAS & $53(100,0)$ \\
\hline $\mathrm{DM}$ & $20(38,0)$ \\
\hline Outras & $7(13,0)$ \\
\hline \multicolumn{2}{|l|}{ Sedentarismo $\mathrm{n}(\%)$} \\
\hline Sim & $48(91,0)$ \\
\hline Não & $5(9,0)$ \\
\hline \multicolumn{2}{|l|}{ Acompanhantes nas AVD n (\%) } \\
\hline Sim & $47(89,0)$ \\
\hline Não & $6(11,0)$ \\
\hline \multicolumn{2}{|l|}{ Tratamento fisioterapêutico $\mathrm{n}(\%)$} \\
\hline Sim & $13(24,5)$ \\
\hline Não & $40(75,5)$ \\
\hline
\end{tabular}

$\mathrm{DP}=$ desvio padrão; $\mathrm{AVE}=$ Acidente Vascular Encefálico; HAS= Hipertensão Arterial Sistêmica; DM= Diabetes Mellitus; $\mathrm{AVD}=$ atividades de vida diária; Alfabetizado $^{\mathrm{a}}=$ ser capaz de ler e escrever pelo menos um bilhete simples no idioma conhecido (Instituto Brasileiro de Geografia e Estatística, Censo de 2007); 1 salário mínimo ${ }^{\mathrm{b}}=510$ reais (medida provisória nº. 474, de 23 de dezembro de 2009). lisando o conjunto de domínios, observou-se que os valores da consistência interna variaram entre $\alpha=0,81$ e 0,87 (Tabela 2 ).

Os resultados da confiabilidade teste-reteste, mostraram que houve uma maior correlação nos domínios "dor", com CCI $=0,92$ (examinador A) e CCI = 0,94 (examinador B); e "habilidades físicas", $\operatorname{com} \mathrm{CCI}=0,92($ examinador $\mathrm{A})$ e CCI $=$ 0,91 (examinador B). Os domínios "nível de energia" e "reações emocionais" obtiveram os menores coeficientes (Tabela 3). Quanto à confiabilidade interexaminadores, os valores do CCI variaram de 0,71 a 0,93 . Conforme pode ser observado pelos valores de $p$, todos os resultados foram estatisticamente significativos (Tabela 3).

$\mathrm{O}$ teste Bland and Altman apresentou a concordância entre as médias obtidas no escore total do PSN em relação ao examinador "A" e entre os examinadores "A" e "B" (Figura 2). Para a concordância teste-reteste, as médias mostraram limites entre $-17,33$ a 21,40 pontos. $\mathrm{Na}$ concordância interexaminadores, os limites variaram entre -19,55 e 25,22 pontos.

\section{Discussão}

A maioria dos indivíduos avaliados era aposentado, chefe de família, com baixa escolaridade e renda familiar per capita $\leq 1 / 2$ salário mínimo ${ }^{17}$. A atribuição de chefe da família vem sendo relacionada à aposentadoria dos que sofreram AVE, os quais passam a ser os provedores da renda familiar ${ }^{18}$. Refere-se ainda que os AVE mais graves e mais incapacitantes ocorrem entre os pertencentes às classes socioeconômicas mais desfavorecidas $^{19}$.

O discreto predomínio de mulheres, embora esteja de acordo com Veloso et al. ${ }^{20}$, difere de outras investigações ${ }^{21,22}$, que relataram predomínio do sexo masculino. Uma revisão sistemática $^{23}$ demonstrou que o AVE é mais comum entre os homens, porém, apresenta-se de forma mais severa nas mulheres. No entanto, devido à perda de autonomia decorrente das incapacidades, independentemente do sexo, o AVE é causa de insatisfação com a vida e de limitações funcionais diversas ${ }^{24}$.

Todos os entrevistados eram portadores de HAS, sendo um dos fatores mais importantes e mais fortemente correlacionados ao $\mathrm{AVE}^{25}$. A Sociedade Brasileira de Hipertensão ainda reforça que a pressão arterial aumenta linearmente com a idade e entre os sedentários o risco de desenvol- 
Tabela 2. Valores médios dos domínios e escore total e a consistência interna do perfil de saúde de Nottingham, quando aplicado em indivíduos pós Acidente Vascular Encefálico, Recife-PE, Brasil, 2010.

\begin{tabular}{|c|c|c|c|c|c|c|c|}
\hline \multirow{2}{*}{ Domínio } & \multirow{2}{*}{ Examinadores } & \multicolumn{3}{|c|}{ Teste 1} & \multicolumn{3}{|c|}{ Teste 2} \\
\hline & & Média (\%) & DP & $\alpha^{\mathrm{a}}$ & Média (\%) & DP & $\alpha^{\mathrm{b}}$ \\
\hline \multirow[t]{2}{*}{ Nível de energia } & A & 65,0 & 36,0 & 0,87 & 73,5 & 31,0 & 0,85 \\
\hline & B & 66,0 & 35,5 & 0,81 & 69,1 & 35,1 & 0,86 \\
\hline \multirow[t]{2}{*}{ Dor } & A & 68,1 & 34,0 & & 68,0 & 33,3 & \\
\hline & B & 65,0 & 33,3 & & 66,5 & 38,0 & \\
\hline \multirow[t]{2}{*}{ Reações emocionais } & A & 68,0 & 27,0 & & 67,0 & 29,1 & \\
\hline & B & 63,3 & 31,1 & & 67,0 & 27,4 & \\
\hline \multirow[t]{2}{*}{ Sono } & A & 69,4 & 32,2 & & 74,0 & 26,0 & \\
\hline & $\mathrm{B}$ & 67,5 & 32,0 & & 70,5 & 35,2 & \\
\hline \multirow[t]{2}{*}{ Interações sociais } & A & 68,3 & 29,4 & & 69,0 & 27,0 & \\
\hline & B & 61,5 & 29,0 & & 67,0 & 26,5 & \\
\hline \multirow[t]{2}{*}{ Habilidades físicas } & A & 50,0 & 24,0 & & 48,0 & 24,4 & \\
\hline & $\mathrm{B}$ & 43,3 & 26,0 & & 41,5 & 24,3 & \\
\hline \multirow[t]{2}{*}{ Total } & A & 64,0 & 23,2 & & 65,0 & 22,1 & \\
\hline & $\mathrm{B}$ & 60,0 & 22,2 & & 62,3 & 24,0 & \\
\hline
\end{tabular}

$\mathrm{DP}=$ desvio padrão; $\alpha^{\mathrm{a}}=$ alfa de Cronbach correspondente ao conjunto de domínios do PSN (teste 1); $\alpha^{\mathrm{b}}=$ alfa de Cronbach correspondente ao conjunto de domínios do PSN (teste 2).

Tabela 3. Confiabilidade teste-reteste e inter-examinadores do questionário PSN, por domínio, em indivíduos pós AVE, Recife-PE, Brasil, 2010.

\begin{tabular}{|c|c|c|}
\hline Comparação & CCI (95\% IC) & Valor de $p$ \\
\hline \multicolumn{3}{|l|}{ Nível de energia } \\
\hline Teste 1 - Teste 2 (examinador A) & $0,77(0,61 ; 0,87)$ & $<0,01$ \\
\hline Teste 1 - Teste 2 (examinador B) & $0,87(0,77 ; 0,92)$ & $<0,01$ \\
\hline Examinador A - Examinador B (teste 1) & $0,82(0,82 ; 0,89)$ & $<0,01$ \\
\hline Examinador A - Examinador B (teste 2) & $0,74(0,55 ; 0,85)$ & $<0,01$ \\
\hline \multicolumn{3}{|l|}{ Dor } \\
\hline Teste 1 - Teste 2 (examinador A) & $0,92(0,86 ; 0,95)$ & $<0,01$ \\
\hline Teste 1 - Teste 2 (examinador B) & $0,94(0,90 ; 0,96)$ & $<0,01$ \\
\hline Examinador A - Examinador B (teste 1) & $0,93(0,89 ; 0,96)$ & $<0,01$ \\
\hline Examinador A - Examinador B (teste 2) & $0,92(0,86 ; 0,95)$ & $<0,01$ \\
\hline \multicolumn{3}{|l|}{ Reações emocionais } \\
\hline Teste 1 - Teste 2 (examinador A) & $0,86(0,75 ; 0,92)$ & $<0,01$ \\
\hline Teste 1 - Teste 2 (examinador B) & $0,83(0,71 ; 0,90)$ & $<0,01$ \\
\hline Examinador A - Examinador B (teste 1) & $0,84(0,73 ; 0,91)$ & $<0,01$ \\
\hline Examinador A - Examinador B (teste 2) & $0,82(0,69 ; 0,89)$ & $<0,01$ \\
\hline \multicolumn{3}{|l|}{ Sono } \\
\hline Teste 1 - Teste 2 (examinador A) & $0,83(0,71 ; 0,90)$ & $<0,01$ \\
\hline Teste 1 - Teste 2 (examinador B) & $0,84(0,72 ; 0,91)$ & $<0,01$ \\
\hline Examinador A - Examinador B (teste 1) & $0,71(0,51 ; 0,83)$ & $<0,01$ \\
\hline Examinador A - Examinador B (teste 2) & $0,82(0,69 ; 0,89)$ & $<0,01$ \\
\hline \multicolumn{3}{|l|}{ Interação social } \\
\hline Teste 1 - Teste 2 (examinador A) & $0,81(0,68 ; 0,89)$ & $<0,01$ \\
\hline Teste 1 - Teste 2 (examinador B) & $0,84(0,73 ; 0,91)$ & $<0,01$ \\
\hline Examinador A - Examinador B (teste 1) & $0,83(0,72 ; 0,90)$ & $<0,01$ \\
\hline Examinador A - Examinador B (teste 2) & $0,79(0,64 ; 0,88)$ & $<0,01$ \\
\hline \multicolumn{3}{|l|}{ Habilidades físicas } \\
\hline Teste 1 - Teste 2 (examinador A) & $0,92(0,86 ; 0,95)$ & $<0,01$ \\
\hline Teste 1 - Teste 2 (examinador B) & $0,91(0,84 ; 0,94)$ & $<0,01$ \\
\hline Examinador A - Examinador B (teste 1) & $0,83(0,70 ; 0,90)$ & $<0,01$ \\
\hline Examinador A - Examinador B (teste 2) & $0,93(0,88 ; 0,96)$ & $<0,01$ \\
\hline
\end{tabular}

PSN= perfil de saúde de Nottingham; AVE= Acidente Vascular Encefálico; CCI= coeficiente de correlação intra-classe; IC= intervalo de confiança. 


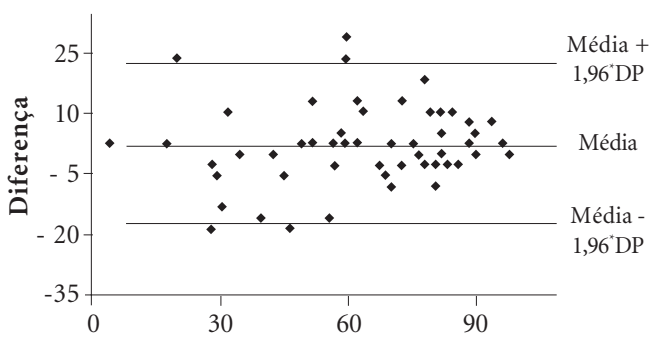

Média do Examinador A (teste 1 - teste 2)\%

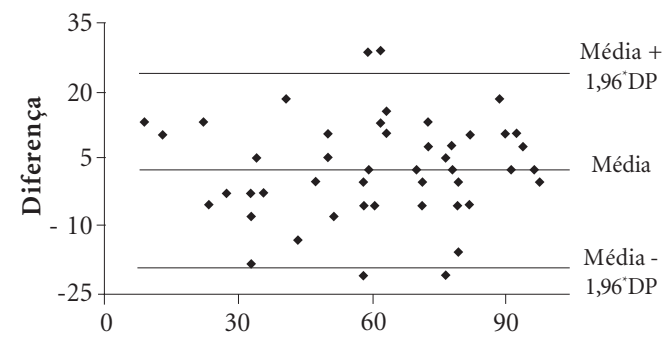

Média (Examinador A - Examinador B)\%

Figura 2. Teste de plotagem Bland and Altman para avaliar a concordância teste-reteste e interexaminadores do escore total do perfil de saúde de Nottingham em indivíduos pós Acidente Vascular Encefálico, Recife-PE, Brasil, 2010.

ver a HAS aumenta em aproximadamente $30 \%{ }^{26}$

Outra característica da amostra foi o discreto predomínio de sequelas neurológicas no hemicorpo esquerdo. Mesmo não havendo um consenso na literatura sobre o dimidio corporal mais frequentemente acometido após o episódio de AVE e embora, para alguns autores, esta informação não pareça ser relevante como fator prognóstico ${ }^{27}$, certamente tem sua importância do ponto de vista clínico e de reabilitação. O AVE é altamente incapacitante e muitos indivíduos permanecem dependentes de algum tipo de ajuda por meses, anos ou mesmo por toda a vida ${ }^{28}$. A diferenciação entre os déficits funcionais de indivíduos com sequelas à esquerda e à direita pode auxiliar no processo de reabilitação e na orientação dos fisioterapeutas quanto à escolha das condutas mais adequadas ${ }^{29}$. No presente estudo, observou-se que uma pequena proporção dos participantes, apenas $24,5 \%$, fazia tratamento fisioterapêutico. Este fato pode ser usado para explicar a grande quantidade de dependentes em suas AVD, realidade também encontrada em comunidades da Cidade de Diamantina, Minas Gerais ${ }^{30}$.

A partir da aplicação do PSN, os resultados apontaram que o domínio HF foi o mais afetado pelo AVE, apresentando a pior percepção de saúde em relação aos outros domínios. Outras pesquisas relacionaram a QV com a prática de atividade física, mostrando que indivíduos ativos têm maior nível de QV quando comparados aos sedentários ${ }^{31,32}$.

Mesmo havendo um prejuízo para o domínio HF, paradoxalmente, o escore total do PSN obteve uma média superior a 50 pontos, demonstrando uma tendência positiva quanto à percepção de QVRS na amostra estudada. Um estudo, avaliando a QV de 153 comunitários com limitações físicas significativas, constatou que 53,4\% dos indivíduos classificaram a sua QV como boa ou excelente, diferindo da opinião pública e até mesmo dos profissionais de saúde que consideraram a QV dessas pessoas como insatisfatória $^{33}$. Portanto, embora possa existir uma relação entre déficits neurológicos e QV, não há necessariamente melhora da percepção de saúde a partir da melhora da função física. Neste sentido, é preciso considerar que as morbidades/comorbidades suscitadas por uma lesão cerebrovascular podem comprometer desde as funções motoras e as sensoriais, a linguagem e até promover alterações emocionais e cognitivas, gerando incapacidades crônicas e impacto negativo na QVRS $^{34}$. Sendo o constructo QV um conceito complexo e multidimensional, sugere-se que os profissionais de saúde não se restrinjam a avaliação apenas de aspectos físicos, sendo relevante, no mínimo, a avaliação dos impactos do AVE também nos domínios mental e social ${ }^{13}$.

O coeficiente á de Cronbach baseia-se no pressuposto de que, ao avaliar uma variável comum, os itens de um instrumento estão positivamente correlacionados uns com os outros ${ }^{17}$. Nesta perspectiva, o PSN apresentou boa consistência interna para a amostra estudada. Outro trabalho, avaliando QV em portadores de doença crônica, obteve resultados semelhantes ${ }^{35}$.

A confiabilidade interexaminadores e do teste-reteste também apresentou valores adequados para todos os domínios avaliados, destacando-se "dor" e "habilidade física", que apresentaram uma correlação excelente $(\alpha>0,90)$. Um estudo conduzido no Japão ${ }^{36}$, em 2004, avaliou 133 doentes crônicos para investigar a confiabili- 
dade teste-reteste do PSN, com intervalo de duas semanas entre as avaliações. Neste, encontrouse confiabilidade acima de 0,85 para o domínio IS. Embora os outros domínios tenham apresentado uma confiabilidade mais baixa, seus valores ficaram acima de 0,70 , portanto, dentro dos limites aceitáveis. Outro trabalho ${ }^{37}$ salientou a importância de reavaliar os dados disponíveis no PSN para melhor analisar seus domínios e, assim, verificar a possível associação com a melhor ou pior percepção de saúde entre os pacientes com doenças crônicas.

O teste de plotagem Bland and Altman mostrou excelente concordância teste-reteste (examinador $\mathrm{A}$ - testes 1 e 2), indicando que as médias obtidas na primeira avaliação estavam concordando com as da segunda em $95 \%$ dos casos. Observou-se também excelente concordância interexaminadores (examinadores A e B - teste 1 ), confirmando que as médias do examinador "A" estavam em concordância com as do examinador "B" em 95\% das ocasiões. Entretanto, mesmo diante dessas evidências, ocorreram alguns casos de outliers no diagrama de dispersão, considerando-se a presença de valores entre os limites extremos dos intervalos de concordância. Guedes et al. ${ }^{38}$, em uma pesquisa que analisou a reprodutibilidade e a validade de um instrumento de avaliação em saúde, afirmaram que os limites de concordância devem ficar o mais próximo possível dos valores equivalentes às diferenças médias. Assim sendo, recomenda-se cautela na interpretação dos dados, quando existir uma variabilidade individual demasiadamente elevada, pois, esta pode influenciar nos valores médios dos escores obtidos nas duas avaliações ${ }^{38}$.

Salvo as limitações inerentes aos estudos transversais, onde não é possível saber se a exposição antecede ou é consequência da doença ${ }^{39}$, acreditamos que, considerando os objetivos do estudo, este desenho foi adequado para identificar as características da QVRS passíveis de intervenção clínica.

Um importante aspecto a ser considerado na presente pesquisa foi a avaliação do estado cognitivo como critério de elegibilidade, minimizando um possível viés de seleção e contribuindo para a validade interna dos resultados. Além disso, o fato do PSN ter sido aplicado por examinadores treinados reduziu as chances de erros na interpretação das perguntas, o que poderia interferir na fidedignidade dos resultados como salientado pelos autores que realizaram a adaptação brasileira ${ }^{8}$.

O PSN, além de ter se mostrado simples e de fácil aplicação, apresentou boa consistência interna para o conjunto dos seus domínios, uma confiabilidade intra e interexaminadores que variou de aceitável a excelente e uma concordância excelente entre as medidas. Com base nestes resultados, a versão brasileira do PSN demonstrou ser um instrumento confiável para avaliar a QV de indivíduos pós-AVE, assim sendo, representa uma alternativa a ser incorporada em protocolos clínicos para o acompanhamento sistemático da percepção de saúde desta população e, na perspectiva de proporcionar eficácia terapêutica, seus resultados são relevantes para o auxílio na decisão clínica.

Diante do exposto, sugerem-se estudos que comparem os resultados do PSN aos de outros instrumentos genéricos amplamente utilizados, como o Short Form 36 Health Survey (SF-36), para avaliar QVRS em portadores de doenças crônicas. 


\section{Colaboradores}

DL Cabral e CG Damascena foram entrevistadoras dos pacientes, digitaram o banco de dados, realizaram a análise e a interpretação dos dados e redigiram o artigo. LF Teixeira-Salmela e GEC Laurentino planejaram e orientaram a investigação, realizaram a revisão crítica relevante do conteúdo intelectual e participaram da aprovação final da versão a ser publicada.

\section{Agradecimentos}

Ao apoio técnico fornecido pelo CAPES - Coordenação de Aperfeiçoamento de Pessoal de Nível Superior, Brasil.

\section{Referências}

1. World Health Organization (WHO). The World Health Report 2002 - Reducing risks, promoting healthy life. Geneva: WHO; 2002.

2. André C. Manual de AVC. 2nd ed. Rio de Janeiro: Revinter; 2006.

3. Dobkin BH. Clinical practice. Rehabilitation after stroke. N Engl J Med 2005; 352(16):1677-1684.

4. Brasil. Ministério da Saúde (MS). Banco de dados do Sistema Único de Saúde. [site na Internet]. [acessado 2010 maio 10]. Disponível em: www.datasus.gov.br/ tabnet/tabnet.htm

5. Makiyama TY, Battisttella LR, Litvoc J, Martins LC. Estudo sobre a qualidade de vida de pacientes hemiplégicos por acidente vascular cerebral e de seus cuidadores. Acta Fisiatr 2004; 11(3):106-109.

6. World Health Organization (WHO). Constitution of the World Health Organization. Basic Documents. Genebra: WHO; 1946.

7. Seidl EMF, Zannon CMLC. Qualidade de vida e saúde: aspectos conceituais e metodológicos. Cad Saude Publica 2004; 20(2):580-588.

8. Teixeira-Salmela LF, Magalhães LC, Souza AC, Lima MC, Lima RCM, Goulart F. Adaptação do Perfil de Saúde de Nottingham: um instrumento simples de avaliação da qualidade de vida. Cad Saude Publica 2004; 20(4):905-14.

9. Lai SM, Perera S, Duncan PW, Bode R. Physical and social functioning after stroke: comparison of the Stroke Impact Scale and Short Form-36. Stroke 2003; 34(2):488-493.

10. Lai SM, Studenski S, Duncan PW, Perera S. Persisting consequences of stroke measured by the Stroke Impact Scale. Stroke 2002; 33(7):1840-1844.

11. Oliveira MR, Orsini M. Escalas de avaliação da qualidade de vida em pacientes brasileiros após acidente vascular encefálico. Rev Neurocienc 2009; 17(3):255-262.

12. Lourenço RA, Veras RP. Mini-exame do estado mental: características psicométricas em idosos ambulatoriais. Rev Saude Publica 2006; 40(4):712-719.

13. Teixeira-Salmela LF, Faria CDCM, Guimarães CQ, Goulart F, Parreira VF, Inácio EP, Alcântara TO. Treinamento físico e destreinamento em hemiplégicos crônicos: impacto na qualidade de vida. Rev Bras Fisioter 2005; 9(3):347-353.

14. Wahlund K, List T, Dworkin S. Temporomandibular disorders in children and adolescents: reliability of a questionnaire, clinical examination, and diagnosis. J Orofacial Pain 1998; 12(1):42-51.

15. Bland JM, Altman DG. Statistical methods for assessing agreement between two methods of clinical measurement. Lancet 1986; 1(8476):307-310.

16. Conselho Nacional de Saúde (CNS). Resolução ${ }^{\circ}$. 196/96. Aprova as diretrizes e normas regulamentadoras de pesquisas envolvendo seres humanos. Diário Oficial da União 1996; 16 out.

17. Pestana MH, Gagueiro JN. Análise de Dados para Ciências Sociais. 4a ed. Lisboa: Síbalo; 2005.

18. Marques S, Rodrigues RAP, Kusumota L. O idoso após acidente vascular cerebral: alterações no relacionamento familiar. Rev Latino-Am Enferm 2006; 14(3):364-371. 
19. Van Den Bos GAM, Smits JPJM, Westert GP, Van Straten A. Socioeconomic variations in the course of stroke: unequal health outcomes, equal care? $J$ Epidemiol Community Health 2002; 56(12):943-948.

20. Veloso F, Reis LA, Azoubel R, Xavier TT, Argolo SM. Um olhar sobre a assistência fisioterapêutica a portadores de acidente vascular encefálico no município de Jequié - BA. Rev Saúde Com 2007; 3(1):5563.

21. Barbosa MAR, Bona SF, Ferraz CLH, Barbosa NMRF, Silva IMC, Ferraz TMBL. Prevalência da hipertensão arterial sistêmica nos pacientes portadores de acidente vascular encefálico, atendidos na emergência de um hospital público terciário. Rev Bras Clin Méd 2009; 7(6):357-360.

22. Duncan P, Studenski S, Richards L, Gollub S, Lai SM, Reker D, Perera S, Yates J, Koch V, Rigler S, Johnson D. Randomized clinical trial of therapeutic exercise in subacute stroke. Stroke 2003; 34(9):2173-2180.

23. Apperlos P, Birgitta S, Andreas T. Sex differences in stroke epidemiology: A systematic review. Stroke 2009; 40(4):1082-1090.

24. Falcão IV, Carvalho EMF, Barreto KML, Lessa FID, Leite VMM. Acidente vascular cerebral precoce: implicações para adultos em idade produtiva atendidos pelo sistema único de saúde. Rev Bras Saúde Matern Infant 2004; 4(1):95-102.

25. Saposnik G, Del Brutto OH. Stroke in South América: a systematic review of incidence, prevalence and stroke subtypes. Stroke 2003; 34(9):2103-2107.

26. Sociedade Brasileira de Cardiologia, Sociedade Brasileira de Hipertensão. V Diretrizes Brasileiras de Hipertensão Arterial. Hipertensão 2006; 9(2):41-77.

27. Macko RF, Ivey FM, Forrester LW, Hanley D, Sorkin JD, Katzel LI, Silver KH, Goldberg AP. Treadmill exercise rehabilitation improves ambulatory function and cardiovascular fitness in patients with chronic stroke: a randomized, controlled trial. Stroke 2005; 36(10):2206-2211.

28. Cruz KCT, Diogo MJD. Avaliação da capacidade funcional de idosos com acidente vascular encefálico. Acta Paul Enferm 2009; 22(5):666-672.

29. Voos MC, Ribeiro do Valle LE. Estudo comparativo entre a relação do hemisfério acometido no acidente vascular encefálico e a evolução funcional em indivíduos destros. Rev Bras Fisioter 2008; 12(2): 113-120.
30. Leite HB, Nunes APN, Corrêa CL. Perfil epidemiológico de pacientes acometidos por acidente vascular encefálico cadastrados na estratégia de saúde da família em Diamantina, MG. Fisioter Pesq 2009; 16(1):34-39.

31. Pacheco M, César MC, Júnior AVO, Storer IA. Qualidade de vida e performance em idoso: estudo comparativo. Saúde em Rev 2005; 7(17):47-52.

32. Rosa F, Geraldo M, Avila M. Qualidade de vida, atividade física e envelhecimento. A Terc Idade 2005; 16:52-65.

33. Albrecht GL, Devlieger PJ. The disability paradox: high quality of life against all odds. Soc Sci Med 1999; 48(8):977-988.

34. Brainin M, Teuschl Y, Kalra L. "Acute treatment and long-term management of stroke in developing countries". Lancet Neurol 2007; 6(6):553-561.

35. Britto RR, Santos CFF, Bueno FF. Reabilitação pulmonar e qualidade de vida dos pacientes portadores de DPOC. Rev Fisioter Univ São Paulo 2002; 9(1):9-16.

36. Nishimura K, Hajiro T, Mckenna SP, Tsukino M, Oga T, Izumi T. Development and psychometric analysis of the Japanese version of the Nottingham Health Profile: Cross-cultural adaptation. Intern Medic 2004; 43(1):35-41.

37. Wann-Hansson C, Hallberg IR, Risberg B, Klevsgård R. A comparison of the Nottingham Health Profile and Short Form 36 Health Survey in patients with chronic lower limb ischaemia in a longitudinal perspective. Health Qual Life Outcomes 2004; 2:9.

38. Guedes DP, Lopes CC, Guedes JERP, Stanganelli LC. Reprodutibilidade e validade do questionário Baecke para avaliação da atividade física habitual em adolescentes. Rev Port Cien Desp 2006; 6(3):265-274.

39. Lima-Costa MF, Barreto SM. Tipos de estudos epidemiológicos: coceitos básicos e aplicações na área do envelhecimento. Epidemiol Serv Saúde 2003; 12(4): 189-201.

Artigo apresentado em 16/03/2011

Aprovado em 24/10/2011

Versão final apresentada em 31/10/2011 